\title{
Possible Depolarization Mechanism due to Low Beta Squeeze
}

\author{
V. H. Ranjbar, Dan Abell \\ Tech-X Corp.
}

A. Luccio, M. Bai

$\mathrm{BNL}$

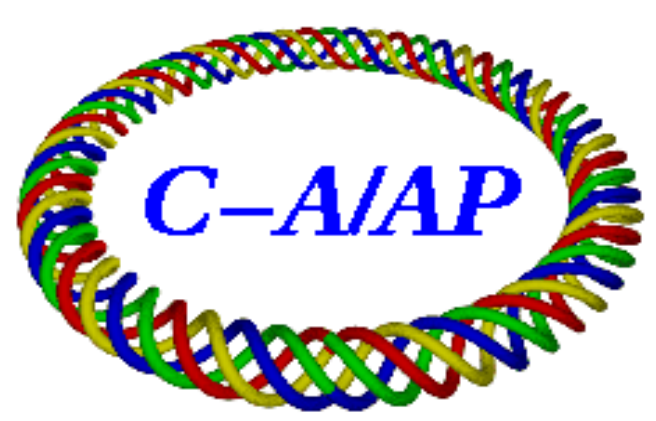

\section{Collider-Accelerator Department Brookhaven National Laboratory Upton, NY 11973}

Notice: This document has been authorized by employees of Brookhaven Science Associates, LLC under Contract No. DE-AC02-98CH10886 with the U.S. Department of Energy. The United States Government retains a nonexclusive, paid-up, irrevocable, world-wide license to publish or reproduce the published form of this document, or allow others to do so, for United States Government purposes. 


\title{
Possible Depolarization Mechanism due to Low Beta Squeeze
}

\author{
V. H. Ranjbar, Dan Abell \\ Tech-X Corp, Boulder Co \\ Alfredo Luccio, Mei Bai \\ BNL, Upton $N Y$ \\ (Dated: March 18, 2008)
}

\begin{abstract}
Simulations reveal a potential depolarization mechanism during low beta squeeze. This depolarization appears to be driven by a spin tune modulation caused by spin precession through the strong low beta quads due to the vertical fields. The modulation of the spin tune introduces an additional snake resonance condition at $\nu_{s 0} \pm n \nu_{x}-\nu_{z} l=$ integer which while the same numerology as the well known sextupole resonance, can operate in the absence of sextupole elements.
\end{abstract}

\section{INTRODUCTION}

Spin effects are tracked employing the TBMT equation,

$$
\frac{d \vec{S}}{d \theta}=\vec{\omega} \times \vec{S}
$$

$\omega$ is usually expressed in terms of an expansion in magnetic field in terms of the Frenet-Serret coordinate system to obtain,

$$
\begin{aligned}
& \omega_{x}=-\rho z^{\prime \prime}(1+G \gamma) \\
& \omega_{s}=(1+G \gamma) z^{\prime}-\rho(1+G)\left(\frac{z}{\rho}\right)^{\prime} \\
& \omega_{z}=-G \gamma+(1+G \gamma) \rho x^{\prime \prime} .
\end{aligned}
$$

Normally to first order the $\rho x^{\prime \prime}$ term is dropped to first order when calculating the spin tune. However if vertical fields are strong enough there can be important contributions to the spin tune via this term. In the presence of snakes this mechanism can introduce a whole new class of snake resonances and allow the horizontal tune to contribute to the snake resonant condition in the absence of linear betatron coupling.

The mechanism is a bit similar to the hybrid resonance first observed in the AGS [1]. We can proceed in a similar fashion by considering the impact on the spin precession of $(1+G \gamma) \rho x^{\prime \prime}$ by integrating,

$$
\int_{0}^{\theta}(1+G \gamma) \rho x^{\prime \prime} d \theta=\left.(1+G \gamma) x^{\prime}\right|_{0} ^{\theta} .
$$

If we consider $x^{\prime}=x_{\beta}^{\prime}$ contributions from only the horizontal betatron motion,

$$
\begin{aligned}
x_{\beta} & =\sqrt{\frac{\epsilon_{N x}}{\pi \gamma} \beta_{x}} \cos \left(\nu_{x} \phi_{x}\right) \\
x_{\beta}^{\prime} & =-\sqrt{\frac{\epsilon_{N x}}{\pi \gamma \beta_{x}}}\left(\sin \left(\nu_{x} \phi_{x}\right)+\alpha_{x} \cos \left(\nu_{x} \phi_{x}\right)\right) .
\end{aligned}
$$

The turn-by-turn perturbation to precession rate sampled at a given location in the lattice $\left(s_{1}\right)$ can now be evaluated using,

$$
\begin{array}{r}
\left.(1+G \gamma) x^{\prime}\right|_{0} ^{N 2 \pi}=(1+G \gamma) \sqrt{\frac{\epsilon_{x}}{\beta\left(s_{1}\right)_{x}}}\left(\sin \left(2 \pi N \nu_{x}+\mu\left(s_{1}\right)_{x}\right)+\alpha\left(s_{1}\right)_{x} \cos \left(2 \pi N \nu_{x}+\mu\left(s_{1}\right)_{x}\right)\right. \\
\left.-\sin \left(\mu\left(s_{1}\right)_{x}\right)-\alpha\left(s_{1}\right)_{x} \cos \left(\mu\left(s_{1}\right)_{x}\right)\right) .
\end{array}
$$

With $N$ being the turn number and $\mu\left(s_{1}\right)_{x}$ the phase at location $s_{1}$. This will introduce sidebands to the spin tune $\nu_{s 0} \pm n \nu_{x}\left(\nu_{s 0}\right.$ the unperturbed spin tune $\left.\nu_{s}\right)$. The effect of these side bands on the spin motion around the vertical 
axis can be estimated by recalling that in the absence of longitudinal and horizontal fields $\left(\omega_{x}=\omega_{s}=0\right)$ the transport of the evolution of the spin vector can be described using,

$$
\begin{aligned}
\frac{d S_{ \pm}}{d \theta} & = \pm i \omega_{z} S_{ \pm} \\
S_{ \pm} & =S_{x} \pm i S_{s} .
\end{aligned}
$$

Thus transport map becomes,

$$
S_{f \pm}=\exp \left(\int_{0}^{\theta} \omega_{z}(\theta) d \theta\right) S_{i \pm}
$$

So from Eq. 5 the Fourier spectrum of $S_{ \pm}$will have a sideband with an amplitude of,

$$
\begin{aligned}
e^{i C} \sum_{m} J_{m}(C) J_{n-m}(S) i^{m} S_{0, \pm} & \\
C & =(1+G \gamma) \sqrt{\frac{\epsilon_{x}}{\beta\left(s_{1}\right)_{x}}}\left(\sin \left(\mu\left(s_{1}\right)_{x}\right)+\alpha\left(s_{1}\right)_{x} \cos \left(\mu\left(s_{1}\right)_{x}\right)\right) \\
S & =(1+G \gamma) \sqrt{\frac{\epsilon_{x}}{\beta\left(s_{1}\right)_{x}}}\left(\cos \left(\mu\left(s_{1}\right)_{x}\right)-\alpha\left(s_{1}\right)_{x} \sin \left(\mu\left(s_{1}\right)_{x}\right)\right)
\end{aligned}
$$

for each $n \nu_{x}$ sideband. This estimation of the strength of the spin tune modulation is valid in the absence of snakes. An analytical estimate of the strength of the spin tune modulation is also possible in the precense of snakes and will be derived. However the location of the frequency remains the same and will in turn alter the snake resonance condition:

$$
\begin{aligned}
\nu_{s}+K l & =\text { integer } \\
\nu_{s 0} \pm n \nu_{x}+K l & =\text { integer } .
\end{aligned}
$$

Which with $K=N \pm \nu_{z}$ the resonant spin tune for an intrinsic resonance, a new hybrid snake resonance condition will appear,

$$
\frac{1}{2} \pm n \nu_{x} \pm \nu_{z} l=\text { integer }
$$

Here $l$ is the order of the snake resonance and $n$ the order of the intrinsic resonance which drives the snake resonance. Experiance with the RHIC lattice has shown that this resonance is important only for $l=1$ and up to $n=2$.

It should be noted that this is the same numerology as the well known sextupole driven snake resonance. The mechanism however doesn't require the existence of sextupoles and is due to 'extra' precession through the quadrupole elements.

This same numerology may apply via a slightly different mechanism. It is also possible that with $B_{x}=B_{s}=0$ that $\omega_{x} \neq 0$. This can be seen by considering the exact expression for $\vec{\omega}$,

$$
\vec{\omega}=(1+G \gamma) \vec{B}-G(\gamma-1) \frac{1}{v^{2}}(\vec{v} \cdot \vec{B}) \vec{v}
$$

Here even with $B_{x}=0$ we will have a nonzero value for $\omega_{x}=-G(\gamma-1) \frac{1}{v^{2}}\left(x^{\prime} z^{\prime} B_{z}\right)$. This second order effect permits an alternate path for the horizontal frequency to drive a snake resonance condition in the absence of betatron coupling.

Spin tune modulation in the presence of snakes but with the remaining ring set to $\omega_{x}=\omega_{s}=0$ can be estimated for the RHIC lattice using the one turn spin transfer map (OTM),

$$
\tau(2 \pi, 0)=t\left(2 \pi, \theta_{s 2}\right) T_{s}\left(\phi_{2}\right) t\left(\theta_{s 2}, \theta_{s 1}\right) T_{s}\left(\phi_{1}\right) t\left(\theta_{s 1}, 0\right)
$$

. In this case $t_{12}=t_{21}=0$ and $t_{22}=t_{11}^{*}=e^{i \frac{1}{2}\left(G \gamma\left(\theta_{f}-\theta_{i}\right)-(1+G \gamma)\left(x^{\prime}\left(\theta_{f}\right)-x^{\prime}\left(\theta_{i}\right)\right)\right)}$. For RHIC's lattice with $\phi_{1}=\frac{3 \pi}{4}$ , $\phi_{2}=\frac{\pi}{4}$ and $\theta_{s 2}-\theta_{s 1}=\pi$. Using the one turn spin transfer map (OTM) we can construct transport map for $S_{ \pm}$ similar to Eq. (7). 


$$
\begin{aligned}
S_{f \pm} & =-S_{i \pm} \exp \left( \pm i\left(2 G \gamma\left(\theta_{s 2}-\theta_{s 1}-\pi\right)+(G \gamma+1)\left(x^{\prime}(2 \pi)-2 x^{\prime}\left(\theta_{s 2}\right)+2 x^{\prime}\left(\theta_{s 1}\right)-x^{\prime}(0)\right)\right)\right) \\
& =-S_{i \pm} \exp \left( \pm i(G \gamma+1)\left(A e^{i 2 \pi Q_{x} n}+A^{*} e^{-i 2 \pi Q_{x} n}\right)\right) \\
& =-S_{i \pm} \exp \left( \pm i(G \gamma+1)|A| \cos \left(2 \pi Q_{x} n+\phi\right)\right) \\
& =-S_{i \pm}\left(\sum_{k=-\infty}^{\infty}(-i)^{k} J_{k}( \pm(1+G \gamma)|A|) e^{i k\left(2 \pi Q_{x} n+\phi\right)}\right)
\end{aligned}
$$

Where $\phi=\arg (A)$ and,

$$
A=\frac{1}{2} \sqrt{\frac{\epsilon_{x}}{\beta_{x}(0)}}\left(1-e^{i 2 \pi Q_{x}}\right)\left[i-\alpha_{x}(0)\right] e^{i \mu_{x}(0)}+\sqrt{\frac{\epsilon_{x}}{\beta_{x}\left(\theta_{s 1}\right)}}\left(-i+\alpha_{x}\left(\theta_{s 1}\right)\right) e^{i \mu_{x}\left(\theta_{s 1}\right)}+\sqrt{\frac{\epsilon_{x}}{\beta_{x}\left(\theta_{s 2}\right)}}\left(i-\alpha_{x}\left(\theta_{s 2}\right)\right) e^{i \mu_{x}\left(\theta_{s 2}\right)}(14)
$$

\section{TRACKING RESULTS}

Using a simplified version of Spink which tracks only the transverse motion, and considers spin precession effects from only, quadrupoles, bends and snakes we have observed depolarization effects which match the resonant condition specified in Eq. 10. The strength of the first order resonant condition appears to be very profound in the current RHIC lattice and observable even with a beta star of $10 \mathrm{~m}$. Examples of this can be seen from tracking results crossing $G \gamma=100-120$ with a normalized vertical and horizontal emittance of $10 \pi$ mm-mrad (see Fig. 1).
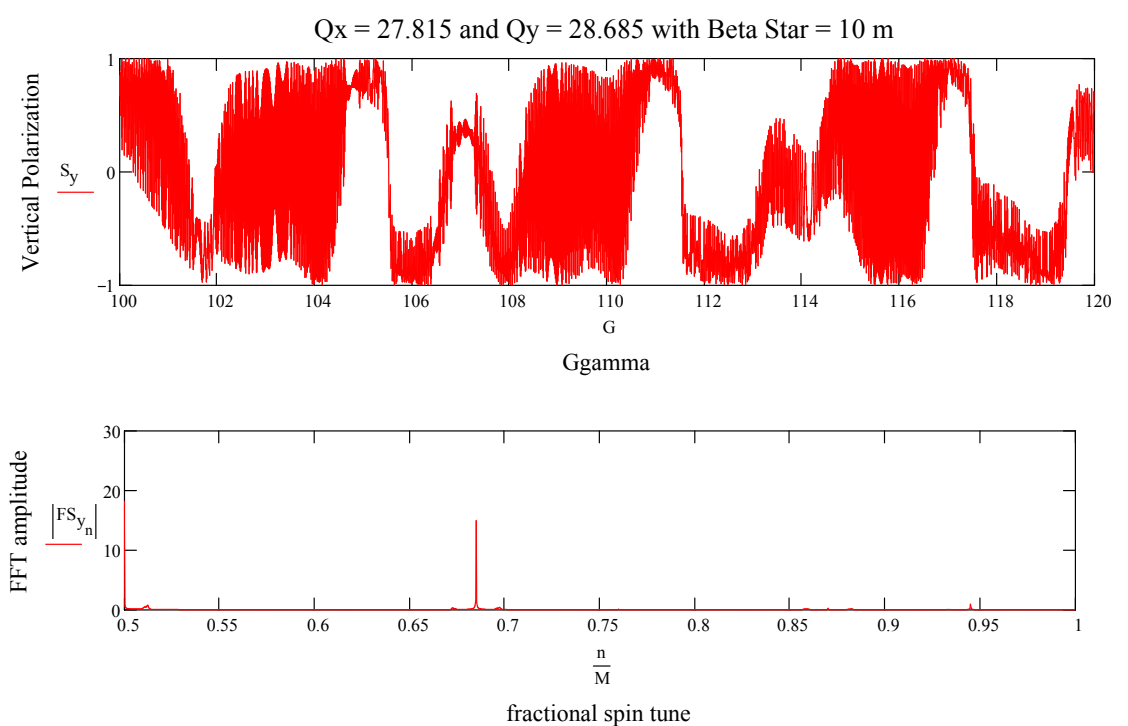

FIG. 1: 1st order hybrid snake resonance. Top plot vertical polarization. Bottom spin tune frequency: note 1st order resonance line at 0.315 and at $0.815+0.5=0.315$.

The strength of the the spin tune perturbation can be compared with our analytical estimate given in Eq. 13 . Running the simulation with the all horizontal and longitudinal frequencies set to zero $\left(\omega_{x}=\omega_{s}=0\right)$ outside the 
snakes. The Fourier spectrum of the $S_{ \pm}$motion can be analyzed at $10 \mathrm{~m}$ beta star and $0.9 \mathrm{~m}$ beta star (see Fig. 2). The ratio of the amplitude of the spin precession frequency strength at $\nu_{s 0} \pm \nu_{x}$ becomes using Eq. (13), $R$ 2.2. Here we used $G \gamma=100$ and a $\epsilon_{N x}=10 \pi m m-m r a d$. This compares with ratio of the Fourier spectrum in Fig. 2 which yields a value of 1.9 .

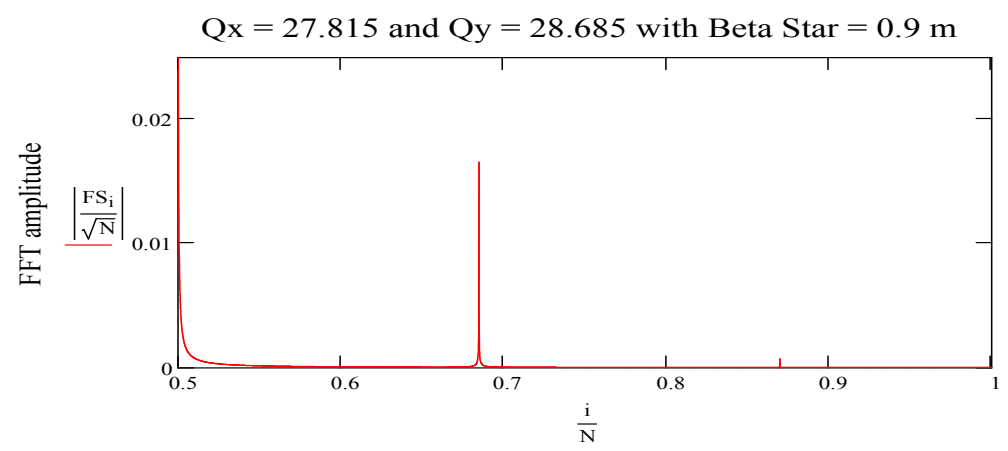

fractional spin tune

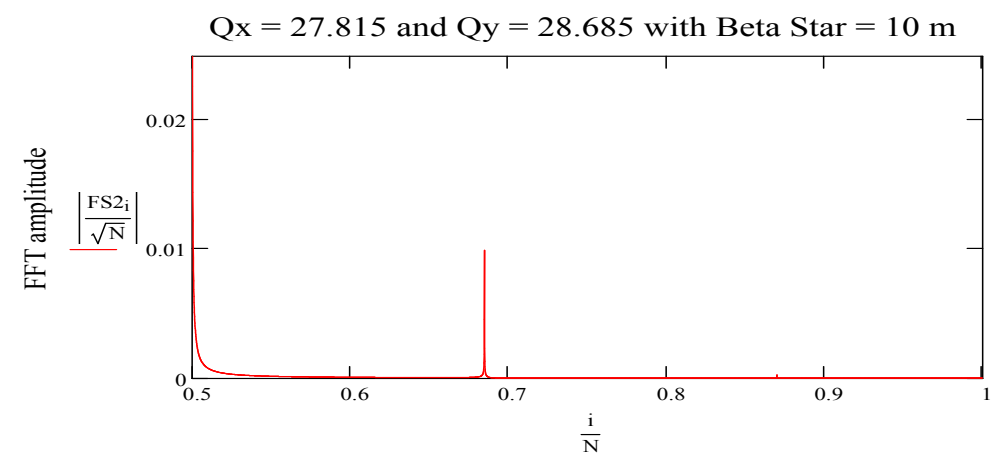

fractional spin tune

FIG. 2: FFT of $S_{ \pm}$for beta star $0.9 \mathrm{~m}$ and $10 \mathrm{~m}$ ratio of freq is 1.9 for freq at 0.685

Interestingly if the $B_{x}=B_{s}=0$ is set to zero only and not $\omega_{x}$ and $\omega_{s}$, we find at this same resonance location significant depolarization occurring even at beta star of $10 \mathrm{~m}$ suggesting that this lattice permits significant significant coupling via the mechanism of Eq. 11.

In addition to the strong first order hybrid snake resonance condition $(n=1)$, the second and third order resonance $(\mathrm{n}=2,3)$ may be important as well for RHIC's current operating tune space and as can be seen in Fig. 3 -4.

Here we can see that a third order $(n=3)$ resonance does not become important until the lattice is squeezed down past $2 \mathrm{~m}$ and one can correlate the depolarization with the strength of the sidebands which together with the strength of the intrinsic resonance drive the depolarization. 


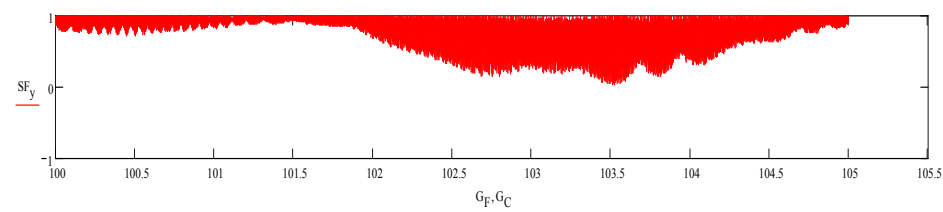

$v x=0.90, v y=0.685$

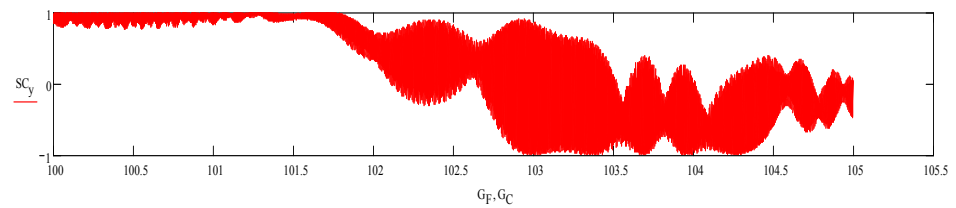

$v x=0.9075, v y=0.685$

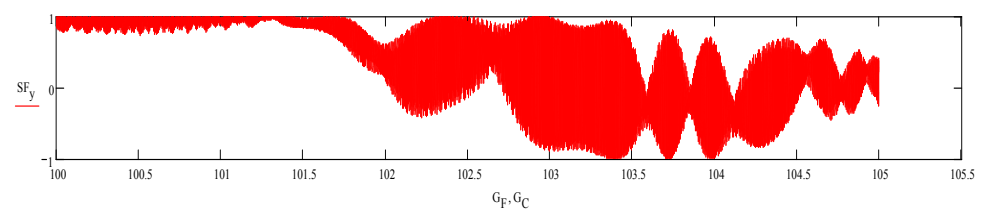

$v x=0.915, v y=0.67$

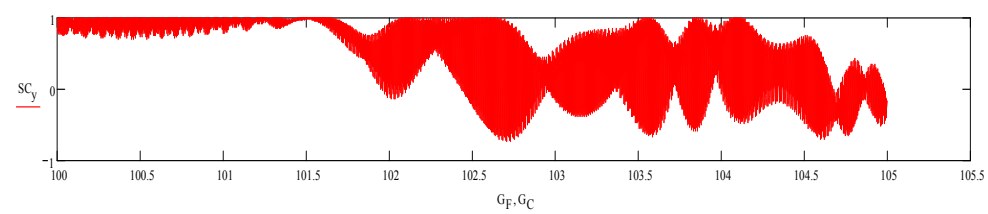

$v x=0.925, v y=0.65$

FIG. 3: Several examples of 2nd order hybrid snake resonance

\section{SIGNIFICANCE FOR RHIC OPERATIONS}

Given the current operating tunes of RHIC ( $\mathrm{Qx}=0.73, \mathrm{Qy}=0.72),(\mathrm{Qx}=0.69, \mathrm{Qy}=0.68),(\mathrm{Qx}=0.893, \mathrm{Qy}=0.886)$, and $(\mathrm{Qx}=0.92, \mathrm{Qy}=0.93)$ this resonance condition is avoided. As can be seen in Fig. 5 tracking from injection to top energy $(G \gamma=45.5-100)$ doesn't seem to reveal any significant depolarization issues.

However this resonance does represent a signifcant reduction of the available tune space. Additionally the asymetric nature of the squeeze in RHIC, squeezing IP6 and IP8 to $0.9 \mathrm{~m}$ and the remaining IP's to $5 \mathrm{~m}$ significantly aliviates some of its effect. For example depolarization for at this second order $(n=2)$ resonance with an aysmetric lattice is typcially about $20 \%$ while a symetric lattice with all IP's squeezed down to $0.9 \mathrm{~m}$ yeilds almost complete depolarization.

[1] M.Bai et. al. "Observation of a Hybrid Spin Resonance" Phys. Rev. Lett. 84, pp 1184-1187 (2000) 


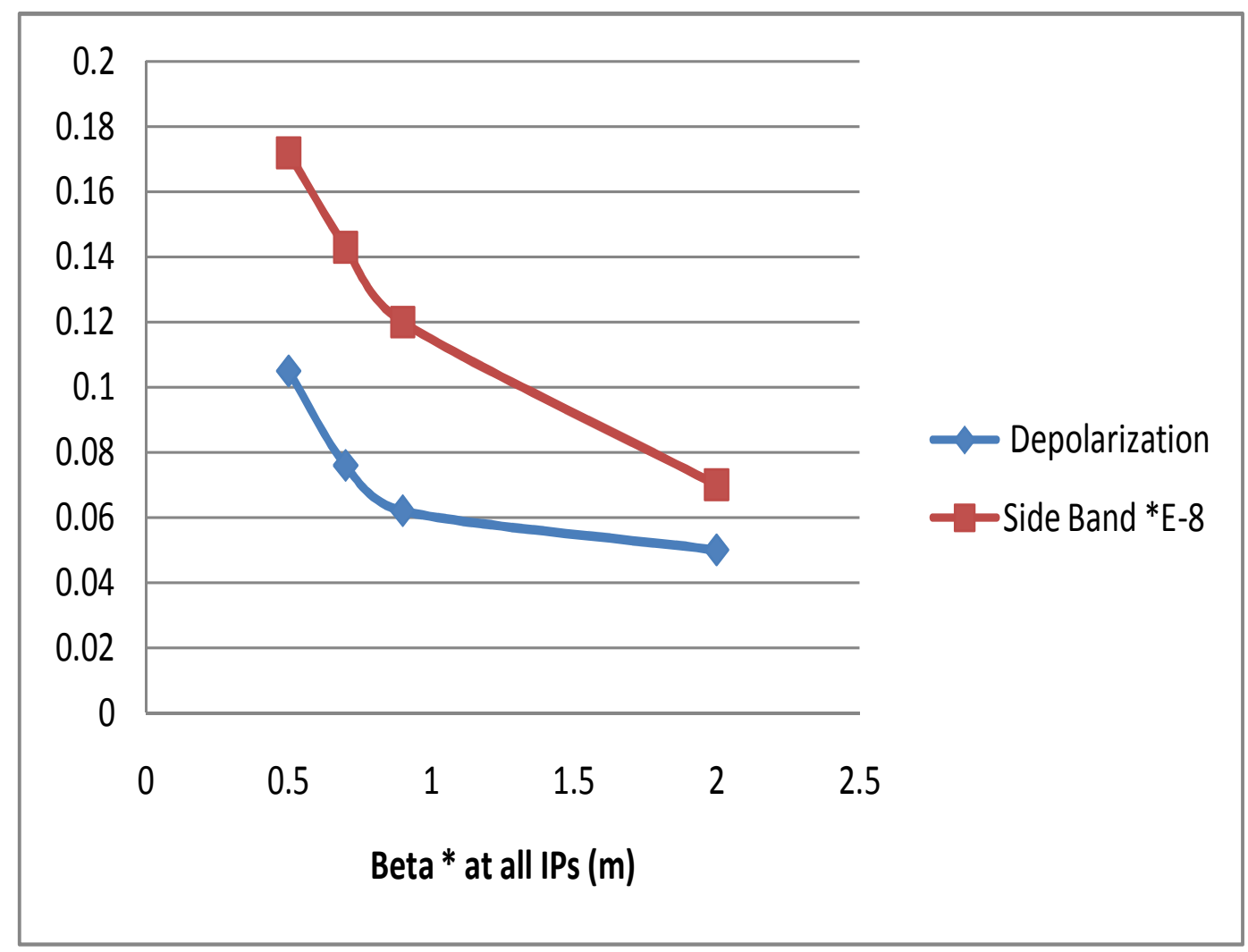

FIG. 4: Polarization response to beta star at 3rd order hybrid snake resonance 

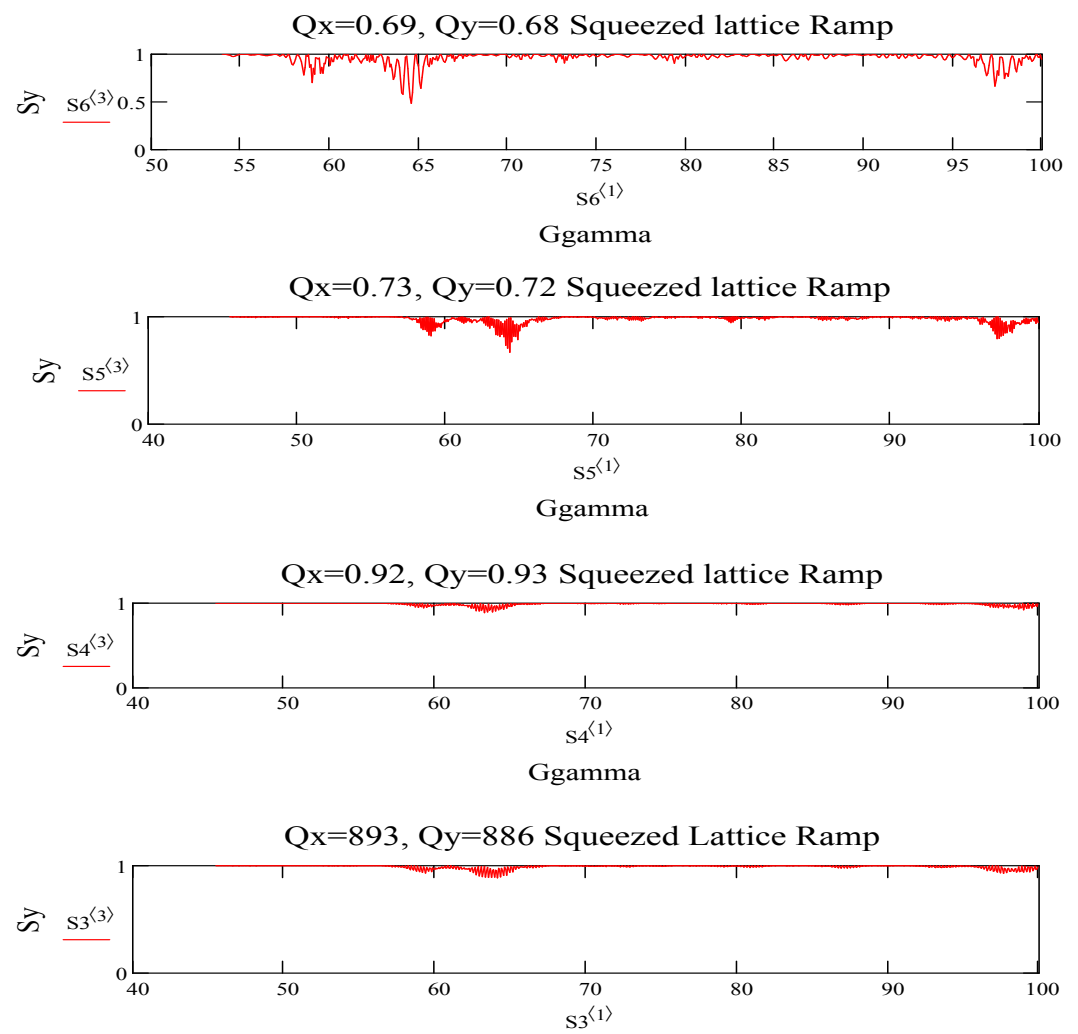

Ggamma

FIG. 5: Single particle tracking result through acceleration ramp at usual RHIC operating tunes with IP 6 and 8 squeezed to $0.9 \mathrm{~m}$ and remaining IP at $5 \mathrm{~m}$ 